\title{
PROOF OF SCOTT'S CONJECTURE
}

\author{
D. SVRTAN
}

ABSTRACr. We give a proof of Conjecture 7 in $[2$, p. 155] first stated in 1881 by R. F. Scott [4]. It reads as follows:

CONJECTURE 7 (R. F. SCOTT). Let $x_{1}, \ldots, x_{n}$ and $y_{1}, \ldots, y_{n}$ be the distinct roots of $x^{n}-1=0$ and of $y^{n}+1=0$, respectively. Let $A$ be the $n \times n$ matrix whose $(i, j)$ entry is $1 /\left(x_{i}-y_{j}\right), i, j=1, \ldots, n$. Then

$$
|\operatorname{per}(A)|= \begin{cases}n(1 \cdot 3 \cdot 5 \cdots(n-2))^{2} / 2^{n}, & \text { if } n \text { is odd, } \\ 0, & \text { if } n \text { is even. }\end{cases}
$$

Actually, our proof gives more, namely an explicit expression for $\operatorname{per}(A)$ (see Theorem 2.1).

1. Preliminary results. Let $A$ be an $n \times n$ matrix $\left(a_{i j}\right)$. The permanent of $A$ is defined by

$$
\operatorname{per}(A)=\sum_{\sigma \in S_{n}} \prod_{i=1}^{n} a_{i \sigma(i)},
$$

where $S_{n}$ is the symmetric group over the set $\{1, \ldots, n\}$.

We first quote two results which we shall use in the course of the proof.

THEOREM 1.1 (BORCHARDT [1]). Let $A$ be an $n \times n$ matrix whose $(i, j)$ entry is $\left(s_{i}-t_{j}\right)^{-1}$, where $s_{i}, t_{j}$ are complex numbers. Then

$$
\operatorname{det}(A) \operatorname{per}(A)=\operatorname{det}\left(A^{(2)}\right) \text {, }
$$

where $A^{(2)}$ has entries $\left(s_{i}-t_{j}\right)^{-2}$.

For the proof see e.g. [2, p. 6]. .

The next result which we need concerns circulants. For given numbers $a_{0}, \ldots, a_{n-1}$ the circulant $C\left(a_{0}, \ldots, a_{n-1}\right)=\left(c_{i j}\right)$ is a $n \times n$ matrix, whose entries are given by

$$
c_{i j}= \begin{cases}a_{j-i}, & \text { if } i \leqslant j, \\ a_{n+j-i}, & \text { if } i>j .\end{cases}
$$

THEOREM 1.2. The determinant of the circulant is given by

$$
\operatorname{det} C\left(a_{0}, \ldots, a_{n-1}\right)=\prod_{k=1}^{n} f\left(x_{k}\right) \text {, }
$$

where $f(t)=\sum_{i=0}^{n-1} a_{i} t^{i}$, and $x_{k}$ are the nth roots of unity.

Received by the editors April 17, 1981.

1980 Mathematics Subject Classification. Primary 15A15, 10A10, 12D05.

Key words and phrases. Permanent, circulant, roots of \pm unity, Viete formulas.

(C)1983 American Mathematical Society 0002-9939/82/0000-0606-\$02.00 
This can be proved easily by multiplying $\operatorname{det} C\left(a_{0}, \ldots, a_{n-1}\right)$ by the Vandermonde determinant $V\left(x_{1}, \ldots, x_{n}\right)$ (cf. [3, pp. 442, 445]).

We shall also need the following properties of the set $H=\left\{h \in \mathbf{C}: h^{n}=-1\right\}$ of the $n$th roots of -1 .

Proposition 1.3. (i) $\sum_{h \in H}(1-h)^{-1}=n / 2$.

(ii) $\sum_{h \in H}(1-h)^{-2}=n(2-n) / 4$.

Proof. Let $h \in H$. Put $x=(1-h)^{-1}$. Then $h=(x-1) / x$ and the following equivalences hold

$$
h^{n}=-1 \Leftrightarrow(x-1)^{n}+x^{n}=0 \Leftrightarrow 2 x^{n}+\sum_{k=1}^{n}(-1)^{k}\left(\begin{array}{l}
n \\
k
\end{array}\right) x^{n-k}=0 .
$$

This enables us to interpret $S=\left\{x=(1-h)^{-1}: h \in H\right\}$ as the set of solutions of the equation (1).

By the Viète formulas we obtain

$$
\sum_{x \in S} x=-(-1)^{1}\left(\begin{array}{l}
n \\
1
\end{array}\right) / 2=n / 2
$$

so that (i) is proved.

To prove (ii) observe that

$$
\sum_{x \in S} x^{2}=\left(\sum_{x \in S} x\right)^{2}-\left(\sum_{x \neq y} x y\right)
$$

Again by Viète formula we obtain

$$
\sum_{x \in S} x^{2}=(n / 2)^{2}-2(-1)^{2}\left(\begin{array}{l}
n \\
2
\end{array}\right) / 2=n(2-n) / 4
$$

thus proving (ii).

\section{The main result.}

THEOREM 2.1. Let $A$ be the $n \times n$ matrix whose $(i, j)$ entry is $\left(x_{i}-y_{j}\right)^{-1}$, where $x_{1}, \ldots, x_{n}$ and $y_{1}, \ldots, y_{n}$ are the distinct roots of $x^{n}-1=0$ and $y^{n}+1=0$ respectively. Then

$$
\operatorname{per}(A)= \begin{cases}(-1)^{[n / 2]} n(1 \cdot 3 \cdot 5 \cdots(n-2))^{2} / 2^{n}, & \text { if } n \text { is odd, } \\ 0, & \text { if } n \text { is even. }\end{cases}
$$

Proof. By Theorem 1.1 we can write

$$
D_{1} \cdot \operatorname{per}(A)=D_{2}
$$

where $D_{\nu}, \nu=1,2$, stands for $\operatorname{det}\left(\left(x_{i}-y_{j}\right)^{-\nu}\right)$.

In order to obtain an evaluation of $D_{\nu}$ 's we first make the following observations.

(a) Let $\varepsilon$ be any $n$th root of -1 . Then the map $g \rightarrow \varepsilon g$ from $G=\left\{g: g^{n}=1\right\}$ to $H=\left\{h: h^{n}=-1\right\}$ is a bijection.

(b) From the definition of the permanent it is readily seen that

$$
\operatorname{per}\left(\left(x_{\sigma(i)}-y_{\tau(j)}\right)^{-1}\right)=\operatorname{per}\left(\left(x_{i}-y_{j}\right)^{-1}\right),
$$

for any $\sigma, \tau \in S_{n}$. 
Therefore, without loss of generality we can assume that the $n$th roots of 1 and -1 are of the form

$$
x_{i}=\alpha^{i}, \quad y_{j}=\varepsilon \alpha^{j}, \quad i, j=1, \ldots, n,
$$

where $\alpha$ is an arbitrary primitive $n$th root of 1 .

By factoring out $x_{i}^{-\nu}$ from the $i$ th row $(i=1, \ldots, n)$ we get

(2) $\quad D_{\nu}=\left(\prod_{i=1}^{n} x_{i}\right)^{-\nu} \cdot \operatorname{det}\left(\left(1-\varepsilon \alpha^{j-i}\right)^{-\nu}\right)=(-1)^{(n-1) \nu} \cdot \operatorname{det} C\left(a_{0}^{\nu}, \ldots, a_{n-1}^{\nu}\right)$

where $a_{k}=\left(1-\varepsilon \alpha^{k}\right)^{-1}, k=0,1, \ldots, n-1$.

Applying Theorem 1.2 to the circulant $C_{\nu}=C\left(a_{0}^{\nu}, \ldots, a_{n-1}^{\nu}\right)$ it follows

$$
\operatorname{det} C_{\nu}=\prod_{k=1}^{n} f_{\nu}\left(x_{k}\right)
$$

where

$$
f_{\nu}(t)=\sum_{i=0}^{n-1} a_{i}^{\nu} t^{i}
$$

For fixed $k, 1 \leqslant k \leqslant n$, we compute

$$
\begin{aligned}
f_{\nu}\left(x_{k}\right) & =\sum_{i=0}^{n-1}\left(1-\varepsilon \alpha^{i}\right)^{-\nu}\left(\alpha^{k}\right)^{i}=\sum_{i=0}^{n-1}\left(1-\varepsilon \alpha^{i}\right)^{-\nu}\left(\alpha^{i}\right)^{k} \\
& =\varepsilon^{-k} \sum_{h \in H}(1-h)^{-\nu} h^{k} \quad(\text { writing } h=1-(1-h)) \\
& =\varepsilon^{-k} \sum_{h \in H} \sum_{j=0}^{k}(-1)^{j}\left(\begin{array}{l}
k \\
j
\end{array}\right)(1-h)^{j-\nu} .
\end{aligned}
$$

Splitting the second sum into two parts given by $j \leqslant \nu-1$ and $j \geqslant \nu$ and using the fact that

$$
\sum_{h \in H} h^{t}= \begin{cases}n, & \text { if } t=0 \\ 0, & \text { if } 0<t<n\end{cases}
$$

we obtain

$$
f_{\nu}\left(x_{k}\right)=\varepsilon^{-k} \sum_{h \in H}\left[\sum_{j=0}^{\nu-1}(-1)^{j}\left(\begin{array}{l}
k \\
j
\end{array}\right)(1-h)^{j-\nu}+\sum_{j=\nu}^{k}(-1)^{j}\left(\begin{array}{l}
k \\
j
\end{array}\right)\right] .
$$

Since $\sum_{j=\nu}^{k}(-1)^{j}\left(\begin{array}{l}k \\ j\end{array}\right)=-\sum_{j=0}^{\nu-1}(-1)^{j}\left(\begin{array}{l}k \\ j\end{array}\right)$, it follows

$$
f_{\nu}\left(x_{k}\right)=\varepsilon^{-k} \sum_{j=0}^{\nu-1}(-1)^{j}\left(\begin{array}{l}
k \\
j
\end{array}\right)\left[\sum_{h \in H}(1-h)^{j-\nu}-n\right] \text {. }
$$

Now, using Proposition 1.3, we get

$$
f_{\nu}\left(x_{k}\right)=\varepsilon^{-k} \begin{cases}-n / 2, & \text { if } \nu=1, \\ \left(\frac{n(2-n)}{4}-n\right)-k\left(\frac{n}{2}-n\right), & \text { if } \nu=2,\end{cases}
$$


or

$$
f_{\nu}\left(x_{k}\right)=\varepsilon^{-k} \begin{cases}-n / 2, & \text { if } \nu=1 \\ -n(n-2 k+2) / 4, & \text { if } \nu=2\end{cases}
$$

Substituting (4) in (3) and (2) we obtain

$$
D_{\nu}= \begin{cases}-\left(\frac{n}{2}\right)^{n} \prod_{k=1}^{n} \varepsilon^{-k}, & \text { if } \nu=1, \\ \left(-\frac{n}{4}\right)^{n} \prod_{k=1}^{n} \varepsilon^{-k}(n-2 k+2), & \text { if } \nu=2 .\end{cases}
$$

From (5) it is clear that $D_{1} \neq 0$. Hence, by (1),

$$
\begin{aligned}
\operatorname{per}(A) & =D_{2} / D_{1} \\
& =(-1)^{n-1} \prod_{k=1}^{n}(n-2 k+2) / 2^{n} \\
& = \begin{cases}(-1)^{[n / 2]} n(1 \cdot 3 \cdots(n-2))^{2} / 2^{n}, & \text { if } n \text { is odd, } \\
0, & \text { if } n \text { is even, }\end{cases}
\end{aligned}
$$

which completes the proof.

As a corollary we have the following identity:

Corollary 2.2.

$$
\sum_{\sigma \in S_{n}} \frac{1}{\prod_{k=1}^{n} \sin (\pi(2 k-2 \sigma(k)+1) / 2 n)}= \begin{cases}n(1 \cdot 3 \cdots(n-2))^{2}, & \text { if } n \text { is odd, } \\ 0, & \text { if } n \text { is even. }\end{cases}
$$

Proof. Put $\alpha=\exp (2 \pi i / n), \varepsilon=\exp (\pi i / n), i=\sqrt{-1}$. Then for the matrix $A$ from Theorem 2.1 we obtain

$$
\operatorname{per} A=(-1)^{n-1} \operatorname{per}\left(\left(1-\varepsilon \cdot \alpha^{k-j}\right)^{-1}\right) \quad(\text { cf. }(2)) \text {. }
$$

Since

$$
\frac{1}{1-\varepsilon \alpha^{k-j}}=\frac{1}{\sin (\pi(2(k-j)+1) / 2 n)} \cdot \frac{i}{2} \exp \left(\frac{2(j-k)-1}{2 n} \pi i\right)
$$

and

$$
\prod_{k=1}^{n} \exp \left(\frac{2(\sigma(k)-k)-1}{2 n} \pi i\right)=-i
$$

for any $\sigma \in S_{n}$, we get

$$
\operatorname{per} A=(-1)^{n} \frac{i^{n+1}}{2^{n}} \operatorname{per}\left(\frac{1}{\sin (\pi(2(k-j)+1) / 2 n)}\right)
$$

Now by Theorem 2.1 the result follows, because the last permanent is precisely equal to the left-hand side of the desired identity.

Note AdDed in Proof. After this article was written (November, 1980) we learnt that Minc (Linear Algebra and Its Applications 28 (1979), 141 - 153, and Kittappa 
(Linear and Multilinear Algebra 10 (1981), 75 - 82) also proved Scott's conjecture. We hope that our proof, which is entirely algebraic and self-contained, is also shorter and more elementary.

\section{REFERENCES}

1. C. W. Borchardt, Bestimmung der symmetrischen Verbindungen vermittelst ihrer erzeugenden Funktion, Monatsb. Akad. Wiss. Berlin 1888 (1855), 165-171; or Crelle's J. 53 (1855), 193-198; or Gesammelte Werke, 97-105.

2. H. Minc, Permanents, Encyclopedia of mathematics and its applications, Vol. 6, Addison-Wesley, Reading, Mass., 1978.

3. T. Muir, Treatise on the theory of determinants, Dover, New York, 1960.

4. R. F. Scott, Mathematical notes, Messenger of Math. 10 (1881), 142-149.

Department of Mathematics, University of Zagreb, 41001 Zagreb, P. O. BoX 187, Yugoslavia

Current address: Department of Mathematics, University of California at Berkeley, Berkeley, California 94720 